\title{
Nouveau certificat de salaire
}

\author{
Un nouveau certificat de salaire (NCS) sera introduit le $1^{\text {er }}$ janvier 2007. Ce sera \\ le document le plus important pour l'imposition fiscale de l'employé et les dé- \\ penses professionnelles qu'il pourra déduire.
}

Guido Schmid

\author{
Correspondance: \\ Guido Schmid, \\ expert-comptable diplômé \\ ABT Fiduciaire \\ Andreas Baumann \& Co. \\ FMH Fiduciaire Services \\ Alte Steinhauserstrasse 1 \\ CH-6330 Cham \\ Tél. 0417486290 \\ Fax 0417486201
}

guido.schmid@fmhtreuhand.ch
L'objectif visé par le NCS consiste essentiellement à augmenter la transparence et à favoriser l'égalité de droit au moyen d'un formulaire uniforme, valable dans toute la Suisse. Différents formulaires sont actuellement en vigueur; ils contiennent des déclarations élaborées il y a des années et ne distinguent pas entre prestations salariales principales et prestations salariales accessoires. En outre, les employeurs remplissent souvent les certificats de salaire de manière divergente.

Le NCS aura aussi pour conséquence l'imposition fiscale de prestations qui n'étaient pas ou que partiellement taxées jusqu'à présent.

Le passage au NCS permettra de détecter les certificats de salaire remplis de manière incomplète par le passé. $\mathrm{Si}$, à cette occasion, les autorités fiscales constatent des erreurs ne résultant pas de négligence ou de l'intention de soustraire intentionnellement certains revenus de l'imposition, elles agiront avec mesure et de manière accommodante, dans les limites admises par la loi.

Compte tenu de la nouvelle transparence des directives, les obligations liées à la déclaration doivent être prises au sérieux. Dans le Guide d'établissement du certificat de salaire, il est indiqué que celui qui ne remplit pas un certificat de salaire ou qui le remplit de manière erronée sera puni (art. 127, 174 et 186 LIFD; art. 43, 55 et 59 LHID; art. 251 Code pénal suisse) et/ou rendu solidaire du montant soustrait (art. 177 LIFD; art. 56 LHID). Le certificat de salaire est en effet un acte officiel.

Le Guide d'établissement du certificat de salaire, édité par la Conférence suisse des impôts (CSI) et l'Administration fédérale des contributions (AFC), décrit les directives formelles en détail. Les anciennes directives ont été reprises dans leur majeure partie, à l'exception des prestations salariales accessoires (fringe benefits) qui doivent désormais être déclarées de manière beaucoup plus détaillée dans les différentes rubriques concernées.

Sont considérées comme prestations salariales accessoires toutes les prestations qui ne sont pas fournies en numéraire ou qui le sont sous forme réduite ou gratuite. Ces prestations doivent être évaluées selon leur valeur marchande ou leur valeur vénale. Voici une sélection de prestations salariales accessoires:

- pension et logement;

- cotisations de rachat pour le $2^{\mathrm{e}}$ pilier;

- droits de participation;

- frais de formation et de perfectionnement;

- véhicule de service utilisé à des fins privées: Le calcul de la valeur de l'utilisation de véhicules de service (part privée) a été fixé de manière uniforme. Si l'employeur assume l'ensemble des coûts, le montant à déclarer s'élève ainsi par mois à $0,8 \%$ du prix d'achat (sans la TVA), toutefois au moins à Fr. 150.- par mois (état septembre 2006). Pour les véhicules en leasing, il faut prendre en compte le prix d'achat au comptant fixé dans le contrat de leasing et non pas le prix d'achat du véhicule (hors TVA). Veuillez toutefois noter que d'autres valeurs de calcul sont momentanément appliquées pour la TVA.

Pour des raisons de praticabilité, il ne faut pas déclarer les prestations suivantes:

- la remise, à titre gratuit, d'un abonnement CFF demi-tarif;

- les réductions en chèques Reka d'une valeur inférieure ou égale à Fr. 600.- par an;

- les cadeaux de Noël, d'anniversaire ou autres cadeaux usuels d'une valeur inférieure ou égale à Fr. 500.- par événement;

- l'utilisation privée d'outils de travail (téléphone portable, ordinateur, etc.);

- la mise à disposition d'une place de stationnement gratuite sur le lieu de travail;

- la remise de miles aériens. Ils doivent être réservés à l'usage professionnel;

- les cotisations d'adhésion à des clubs ou associations inférieures ou égales à Fr. 1000.par adhésion. Si elles dépassent ce montant, le montant total devra être indiqué;

- les cotisations annuelles aux associations professionnelles, quel que soit leur montant; 
- le montant total des frais de formation et de perfectionnement, lorsqu'ils sont directement versés par l'employeur et qu'ils ne dépassent pas le montant de Fr. 12000.- par année et par collaborateur;

- etc.

La déclaration des allocations pour frais contient également une nouveauté importante, à savoir la distinction entre remboursement des frais effectifs sur présentation de justificatifs, allocations forfaitaires pour frais versées pour une période déterminée et allocations pour frais versées dans le cadre d'un règlement des remboursements de frais approuvé par les autorités fiscales.

Il faudra donc définir les frais de manière à ce qu'ils puissent ensuite être déclarés facilement. Nous recommandons de les fixer dans un règlement concernant les frais qui devra être soumis aux autorités fiscales pour approbation. Les règlements agréés présentent l'avantage de dispenser l'entreprise de déclarer les frais effectifs au moyen de montants détaillés. Nous vous conseillerons volontiers pour l'élaboration d'un règlement et la procédure d'approbation le concernant.
Nous vous recommandons de procéder comme il suit afin d'éviter toute surprise désagréable:

- introduire des structures salariales simples et faciles à comprendre ou effectuer les adaptations nécessaires;

- éviter tout frais à caractère salarial;

- vérifier/adapter/introduire le règlement concernant les frais;

- faire approuver le règlement concernant les frais;

- définir clairement toutes les prestations salariales accessoires et les fixer par écrit;

- le cas échéant, adapter les contrats de travail;

- adapter l'informatique;

- adapter les données salariales de base;

- envisager l'externalisation de la comptabilité salariale à une fiduciaire.

Vos partenaires de confiance de FMH Fiduciaire Services se tiennent volontiers et en tout temps à votre disposition pour répondre à vos questions. 